\title{
Development of User-Interactive Online Education System using Web-based Computational Science Simulation Platform
}

\author{
Gi-Myeong Ryu ${ }^{1}$, Jung-Hun $\mathrm{Shin}^{2}$, Sik Lee ${ }^{3}$ and Kum-Won $\mathrm{Cho}^{4}$ \\ ${ }^{1234}$ Korea Institute of Science and Technology Information \\ $\left\{{ }^{1}\right.$ symsonic, ${ }^{2}$ shandy77, ${ }^{3}$ siklee, $\left.{ }^{4} \mathrm{ckw}\right\}$ @ kisti.re.kr
}

\begin{abstract}
Following the introduction of a Web-based computational science platform Known as the EDucation-research-industry Integration through Simulation On the Net (EDISON), in 2011, approximately 10,000 users annually attended university lectures in Korea. EDISON massive open online courses (MOOCs) were developed in the field of computational fluid dynamics by using the advantage of online free public lecture in order to embody the function of differentiated engineering education in the existing EDISON platform. It is structured to provide e-learning contents such as quizzes and response lecture materials, which are different from the unilateral teaching methods of a regular teacher, so that students can improve their concentration and comprehension. The most important feature is that it is possible to link EDISON's simulation environment with EDISON MOOC to provide an interactive environment for experimentally visualizing analysis result files directly in the lecture screen or interactively controlling $3 D$ grid files. EDISON MOOC is expected to contribute to the qualitative growth of engineering education by shifting from lectures focused on professors to creative and active learning centered on learners
\end{abstract}

Keywords: web-based computational science platform, EDISON MOOC, computational fluid dynamics, interactive environment, e-learning contents

\section{Introduction}

The need for a convergence platform is a challenging issue. The platform should respond to rapidly changing technology such that it can be used for education, research, and industry in computational science and engineering as opposed to high-risk and highcost experiments. The national research foundation in Korea provides a web portal known as EDucation-research-industry Integration through Simulation On the Net (EDISON), for education and research in professional application fields such as computational fluid dynamics, computational nanophysics, computational chemistry, computational structural dynamics, computer-aided optimal design, and computational medicine. The EDISON platform contributes to nurturing advanced and practical human resources that can be immediately applied in R \& D and industrial fields, and it is improves national prestige by leading future education and its global science technology. Additionally, we replace the foreign software (SW) in the domestic education and research SW market by acquiring core technologies education, research, and industrial simulation SW in the field of science and engineering as well as Science App Store core technology. The EDISON platform consists of a system that enables researchers and university students in computational science and engineering to connect to the EDISON Portal and visually conduct multidisciplinary and applied principles in real time for research, learning, and virtual experiments. It is developed as a platform that is used for education, research, and industrial use in a cyber environment by integrating the latest research achievements in

Received (November 26, 2017), Review Result (January 26, 2018), Accepted (February 1, 2018) 
various specialized fields to construct an engineering ecosystem with advanced information technology. It is composed of an application framework, a middleware, and an infrastructure to support multidisciplinary scientific engineering field. It also includes technologies including the Science App Engine, workflow execution engine optimization, resource and task management, data storage, and data-driven simulation services. The computational science, engineering education, and research convergence platform (EDISON) was formally launched in 2011, and it provides web-based simulation services to students and researchers based on 413 SWs and 671 contents. As of September 2017, 48,952 students in EDISON were studying 1,382 subjects in 455 colleges [1]. The recognition of engineering education is gradually increasing with increase in the number of users that utilize the web-based computational science engineering platform. EDISON makes it easier to understand complex mathematical formulas and difficult scientific theories through simulation. However, the absence of specific background theory and the lack of explanation of the link between numerical techniques and the functioning of the tool hinder accurate user learning. This evolves into a problem of focusing on the use of the tool as opposed to the theoretical basis of a highly scientific system in terms of students.

In this study, we propose a user-responsive online education environment that includes an authoring tool function that grasps the theoretical basis of numerical analysis methods, which is difficult for users based on computational science simulation service fundamentally provided on the EDISON platform. Specifically, theoretical lectures and practical exercises are combined in the field of fluid analysis, such that the interactive learning space is aimed at increasing the learning effect, and it is possible to select and learn only the lectures through the lectures classified based on the subjects. It is expected to constitute an innovative engineering education method that allows students to actively reduce the gaps between theoretical and actual physical phenomena.

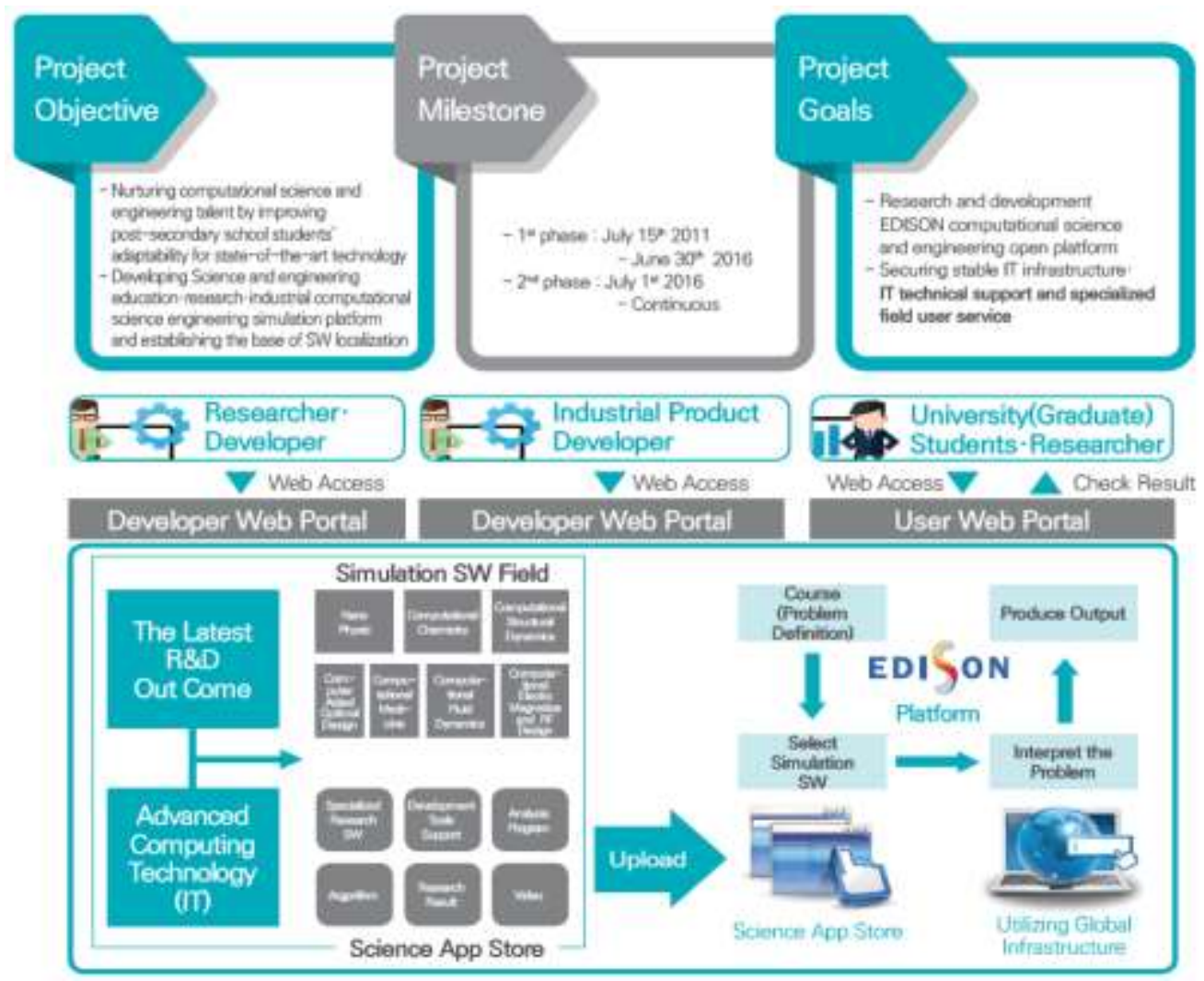

Figure 1. EDISON Project Objective and Goals 


\subsection{Portal Service Layer}

We built a web portal framework for simulations used for education and research by automatically loading the simulation SW and contents developed by domestic and international researchers in computational science and engineering. It includes six types of new themes and 15 technologies including the Science App Store, simulation, virtual class, advanced content portlet, and pre/post processing portlet. It supports the integrated management of user data for multidisciplinary convergence research and provides full management server redundancy service by considering both performance and sustainability. It also improves platform compatibility with cross-browsing web standards sites and maintains forums and bug tracking systems to address platform requirements and other site issues. As shown in Figure 2, these portal services are responsible for the connection and integration of six platforms in computational science and engineering (for six fields as follows: computational fluid dynamics, computational nanophysics, computational chemistry, computational structural dynamics, computer-aided optimal design, and computational medicine).
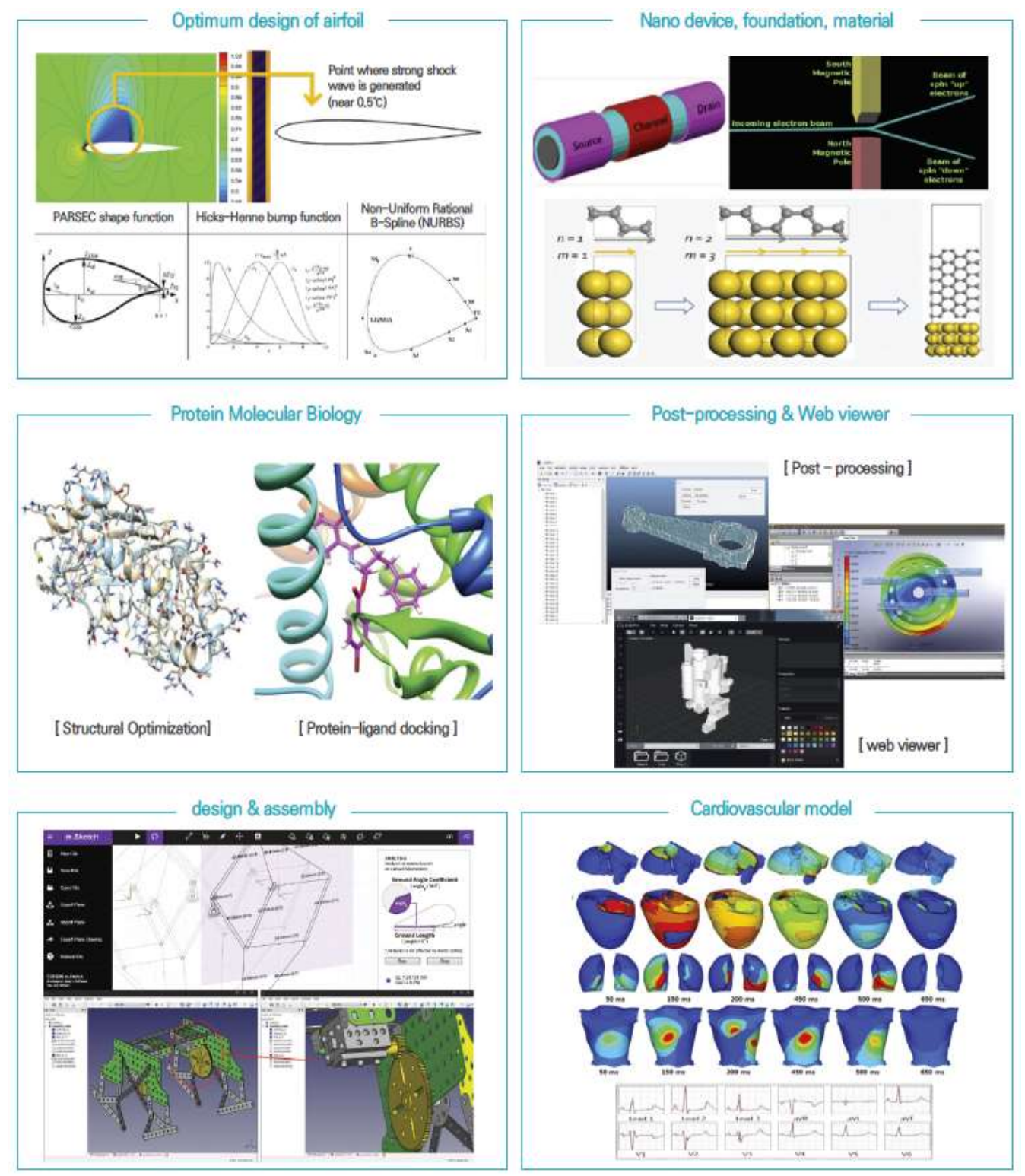

Figure 2. Six Platforms in Computational Science and Engineering 


\subsection{Middleware Layer}

The middleware provides a computational science big data processing framework and a supercomputing-based execution framework. We developed detailed metadata collection, preservation, statistics, analysis, and mining techniques. Additionally, SW meta information management technology, heterogeneous computing resource management technology, workflow management, and execution technology was developed. The workflow actor management technology, workbench, and contents manager were developed. These tools occupy pivotal roles in the EDISON platform. Specifically, the workflow actor management technology extends the Science App Store. The workbench operates to create, manage, and execute workflows. The contents manager copes with the responsive web content.

\subsection{Infrastructure Layer}

EDISON provides a total of 4664 cores to compute resources and $162.9 \mathrm{~TB}$ of storage for data services. Additionally, various monitoring environments are supported to use automatic problem diagnosis and notification service, regarding system and simulation, real-time service utilization trends, fault status, and infrastructure resource utilization.

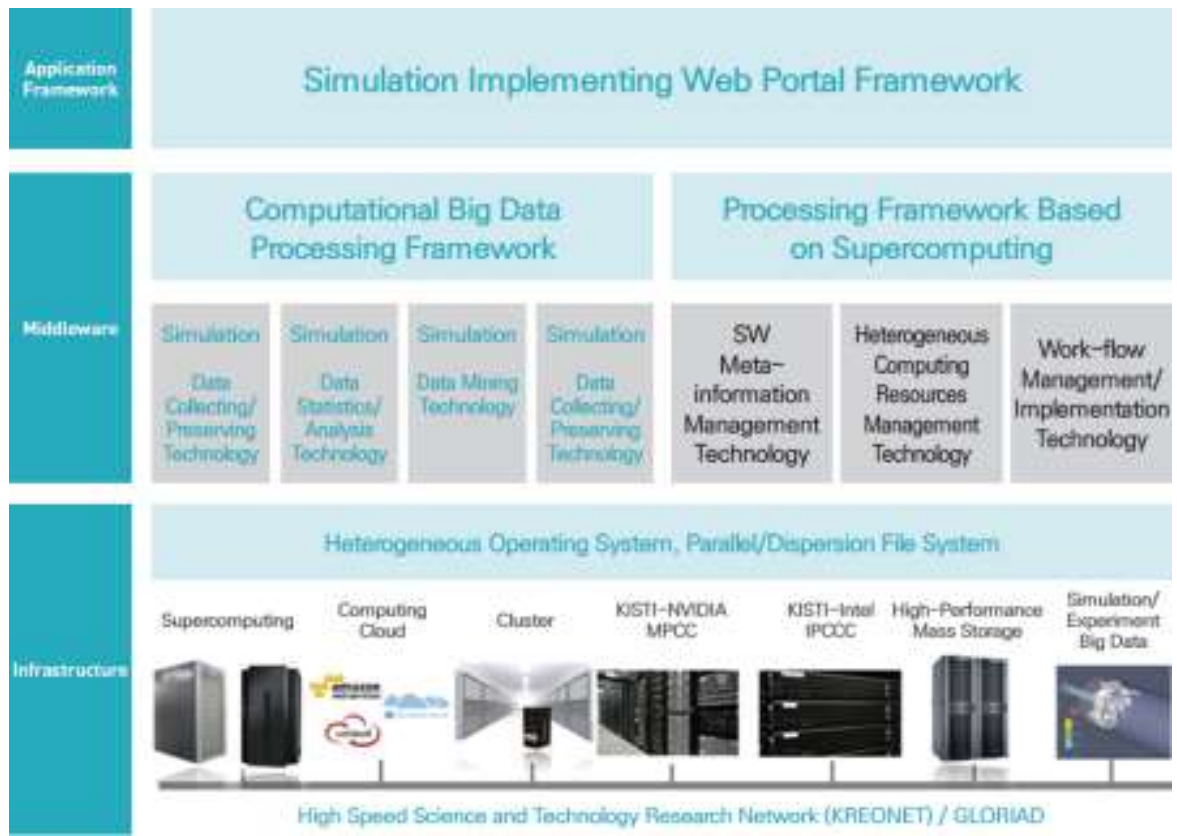

Figure 3. EDISON Open Platform

\section{Educational Uses of EDISON}

From 2011 to date, the Korea Institute of Science and Technology Information (KISTI) conducted 1,237 training sessions on university lectures by using the EDISON platform, and 562 papers were presented through six competition events [2]. These use cases were already demonstrated in nanoHUB. In nanoHUB, 185 institutions, 1,500 classes, and more than 29,910 students used simulation tools in the field of nanotechnology [3]. However, the field is limited and lacks academic exchanges and competition exhibition. The EDISON platform is equipped with simulation software and contents developed by researchers of science and engineering universities in six domestic fields and is used by more than 100 universities and colleges in science and engineering universities nationwide with online access. Additionally, undergraduate and graduate students of universities nationwide publish articles on various research themes by using EDISON 
platform and SW, and a total of 562 teams and 1,178 students competed in the computational science simulation analysis ability until the 6th competitive exhibition.

\subsection{Use in Lecture-Based Education}

Universities use the EDISON platform to promote the creative thinking and problemsolving skills of science and engineering students. The EDISON platform provides practical and experiential web-based education services through various simulation tools and educational contents as opposed to previous theoretical education methods. Additionally, an increase in the degree of understanding is expected by easily accessing the high-performance free software that performs the same functions as opposed to expensive foreign software that students experience difficulties in accessing.

2.1.1. Computational Fluid Dynamics (CFD): As the center of an education group for CFD, universities with departments including aeronautical, mechanical, civil engineering, and design use the EDISON platform as an educational tool in numerical analysis, fluid mechanics, and aerodynamics. Instructors conduct open lectures on the web portal. Undergraduates and graduate students attend lectures in the courses by analyzing the phenomena of fluid physics and performing numerical calculations to directly model them.

2.1.2. Computational Nanophysics (NANO): As the center of an education group for NANO, universities with departments including physics, electronics engineering, and materials engineering use the EDISON platform as an educational tool for visualizing concepts including general physics, mechanics, electromagnetics, semiconductor devices, and materials engineering in classes to help students access the same easily. It further aids the students submit assignments and encouraging the use of simulation tools in the assignments.

2.1.3. Computational Chemistry (CHEM): As the center of an education group for CHEM, universities with departments including quantum chemistry and statistical thermodynamics use the EDISON platform for molecular design, calculation, and/or analysis tools for computational chemistry simulations. Experimental lessons are conducted through the EDISON computational chemistry sites while theoretical lessons are transmitted between instructors and students via the education sites of the EDISON web portal. Thus, the EDISON computational chemistry platform complements theoretical and experimental lessons. KISTI's supercomputing resources provide an indepth interpretation, and high-quality information is provided to students attending courses through EDISON.

2.1.4. Computational Structural Dynamics (CSD): As the center of an education group for CSD, universities with departments including mechanical, automotive, civil engineering, and aerospace use EDISON platform to focus on the engineering disciplines of structural analysis to analyze thermal stress, deformation, and aero elasticity.

2.1.5. Computational Aided Optional Design (DESIGN): The EDISON Designer is used as a teaching tool for topics including computer aided design (CAD), computer aided engineering (CAE), and Optimum Design focusing on flow/structure related university/graduate school classes in conjunction with the field of computational fluid and structural dynamics. Instructors design courses regarding modeling shapes, CAE, and optimization. Subsequently, students design the structure by using open-source based CAD software. This decreases the dependency of expensive foreign software while increasing the market share of the domestic software, and thereby decreases the burden on industry-academia-research institutes. 
2.1.6. Computational Medicine (CMED): EDISON computational medicine tools are used to educate the various functions of the human body around universities with related departments including physiology, basic/clinical pharmacology, and biomedical engineering. Based on the lectures created by the instructor, the students attend lectures, and the simulations provide the synergy effect of the theory and practice and the substitution effect of animal training.

\subsection{Use in competition exhibition}

To improve students' creative thinking and problem-solving abilities of the science and engineering students and to revitalize the community, we annually organize SW competitions to promote SW promotion, secure users, and expand the base. Additionally, several students in the competition verify the stability of the software and the reliability of the results by contacting and executing the software online.

2.2.1. Computational Fluid Dynamics (CFD): The CFD Competition is held annually in conjunction with the Korea Computational Fluid Engineering Association (KSCFE). Students select the topics of their choice, use the fluid analysis software provided by the EDISON platform to solve the problems, and write meaningful analysis results and findings in the form of a paper.

2.2.2. Computational Nanophysics (NANO): The use of SW in the field of nano/materials is held annually in conjunction with the Korean Physical Society (KPS). For example, the semiconducting problem constitutes an opened issue via nanosimulation tools, and a number of excellent papers by using these tools are published to expand the base of nanophysics SW. Participants in the competition submit their papers via the EDISON web portal.

2.2.3. Computational Chemistry (CHEM): The competition is held annually in conjunction with KCS (Korean Chemical Society), by using the EDISON computational chemistry system, and posters or evaluation of oral presentation will be presented. Awards of excellence are presented. It shows that the developed programs exhibit excellent competitiveness based on excellent research results through competition.

2.2.4. Computational Structural Dynamics (CSD): The EDISON Structural Dynamics Competition is held annually and aims to expand the supply of advanced science and technology in Korea through academic motivation and literacy in the latest fields of science and technology. Participants of the competition solve the problems related to free topics through the EDISON structural static analysis program, write articles, and generate excellent research results.

2.2.5. Computational Aided Optional Design (DESIGN): The EDISON ComputerAided Optimal Design Competition is held annually for the purpose of establishing a virtuous cycle of university student education and research through linkages with the CDE Society of Korea. Participants demonstrate their superiority through computerized design of Jansen mechanism walking robots running games and paper presentations.

2.2.6. Computational Medicine (CMED): This program is held annually once a year in conjunction with the Korean Physiological Society (KPS) to solve problems related to computational medicine by presenting research papers. The results of using the EDISON computational medicine simulation in physiology, biomechanics, and pharmacology are presented and submitted. 


\section{EDISON MOOC}

Currently EDISON's educational system is simulated by creating virtual classes in computer science and engineering college courses nationwide [4]. Students are connected to the web environment with their personal IDs. Students perform simulations, and visualize the results. However, in actual theoretical classes, most lectures are taught as per conventional methods. Only practical lessons are connected to the virtual class of the EDISON platform for simulation analysis. Although several manuals are used in practical lessons, there is a limited range of background for theory explanations or for using the tools. Thus, it is necessary for students to experience the difference between theoretical and practical lessons. Additionally, students who are new to the EDISON system are curious about the use of specific functions and their relation to the physical phenomenon. Currently EDISON's education system relies only on the ability of teaching assistants to teach lectures.

\subsection{User Satisfaction Survey for Current EDISON's Educational System}

In EDISON Central Center, we conducted surveys involving 35 universities, 244 lectures and 5,232 students (58.2\%, total number of users: 8,993 students) from July 1, 2016 to June 30, 2017 to investigate and analyze the satisfaction and requirements of portal users. Surveys were conducted online through virtual class or contest questionnaire pages. The questionnaire consisted of 13 questions (10 multiple choice questions and 3 short answers pertaining to satisfaction related to topics including web portal use, satisfaction with simulation environment, and satisfaction with manual and lecture composition) for service improvement. Among these, the user's requirements for online education included video lectures, detailed manuals, and lecture scheduling for teaching assistants.

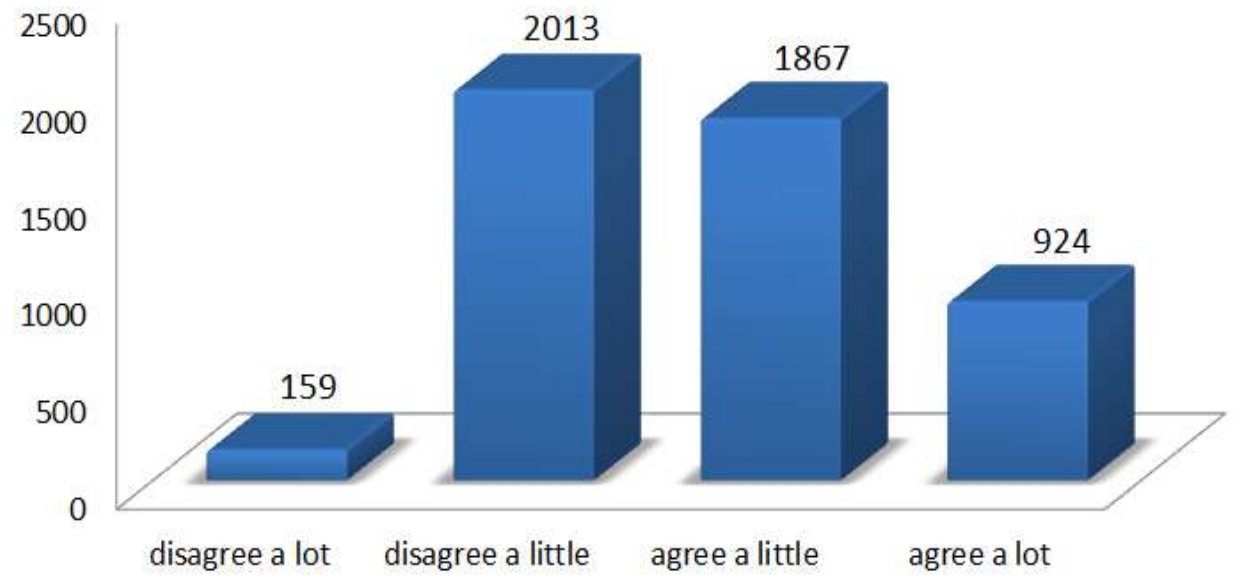

Figure 4. EDISON's User Manual and Lecture Notes are Easy to Understand

As shown in Figure 4, EDISON manuals and lecture materials are sufficient for students to understand the lessons. However, $43.8 \%$ of the respondents indicated that the manuals and lecture materials were insufficient. This is due to the fact that the ratio of first and second year undergraduates among all users accounts for $62.9 \%$ of the total students and is significantly influenced by the difficulties of using computational sciences and numerical analysis theory and tools that are difficult for lower grade students to 
understand. The analysis indicated that it is necessary to focus on more detailed lecture explanations and detailed manuals.

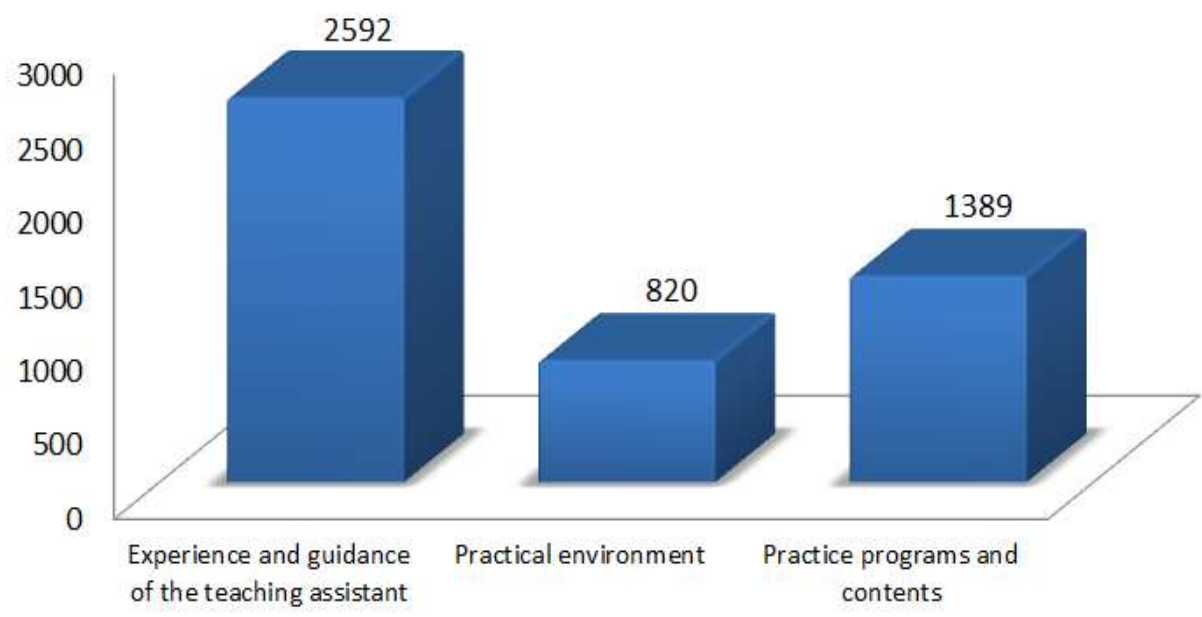

Figure 5. Most Important Factor in Virtual Class by using the EDISON Platform

Figure. 5 shows a very interesting answer in which the most abundant experience and teaching abilities of assistant professors are selected as an important factor in practical training. This is mainly because the lower grade students use EDISON in college lectures, and thus, they often use the first computational science engineering simulation program. It is not easy to use manuals and lectures to fully understand aspects including the meaning and use of a new program, how it is used in the actual engineering field, and what the result of a simulation means. Therefore, a skilled assistant initially instructs students on the meaning of each practice or a simple theory. Thus, the instruction of a skilled assistant with respect to the physicochemical meaning of the simulation result is a very important factor.

\subsection{Need for User-Interactive Online Training}

In the field of computational science and engineering, the introduction of online education, such as Massive Open Online Courses (MOOC), is extremely important. However, in the existing MOOC, the learner's experience corresponds to indirect experience through the web as opposed to direct experience. Learners achieve authentic learning by direct experience and not indirect experience, and thus direct experience in education is very important [5]. With respect to the EDISON platform, a direct experiential learning fortunately exists through simulation exercise. Additionally, with respect to the existing virtual classes, MOOC in the computational science field provides a wealth of manual and lecture materials and interactive e-learning contents to enhance learning motivation and understanding. In MOOC, the learning effect is expected to increase if direct and active experience such as simulation analysis process is performed by users while directly handling the tools.

\subsection{Features of EDISON MOOC}

The MOOC provided by EDISON is limited to the computational fluid dynamics field and serves as a pilot. As shown in Figure 6, the curriculum is divided into individual learning objects. A total of 587 lectures and learning elements are constructed, such that learners selectively choose lectures by grades, preferences, and levels. 


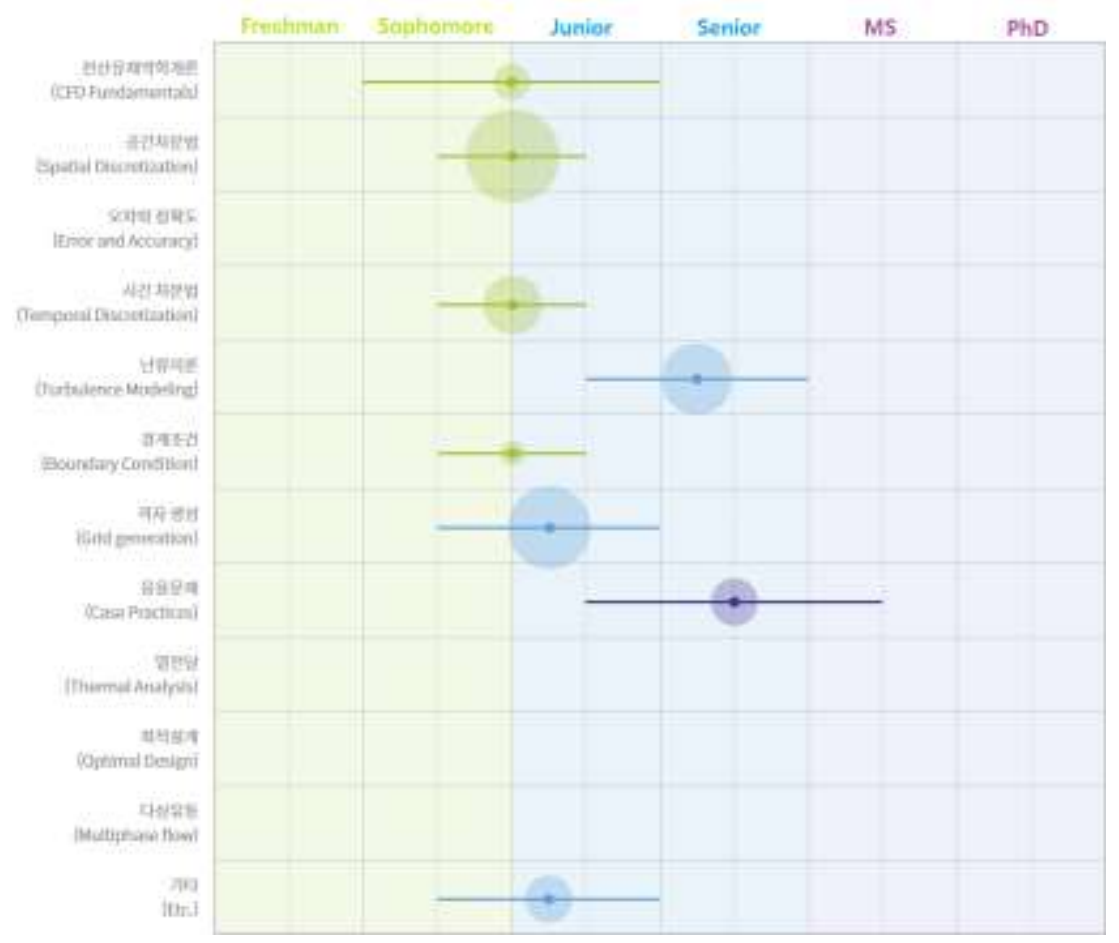

Figure 6. EDISON MOOC Curriculum Map

Additionally, the Course Builder authoring tool is built to enable teachers to create new online courses. It is provided as an Open Learning XML (OLX) method that conforms to international standards and Korean Massive Open Online Course (KMOOC) such that it is not dependent on a specific system. Additionally, as shown in Figure 7, authoring tools that were developed into AngularJS by using various types of media were provided. These tools continuously produce rich educational content and online courses [6].

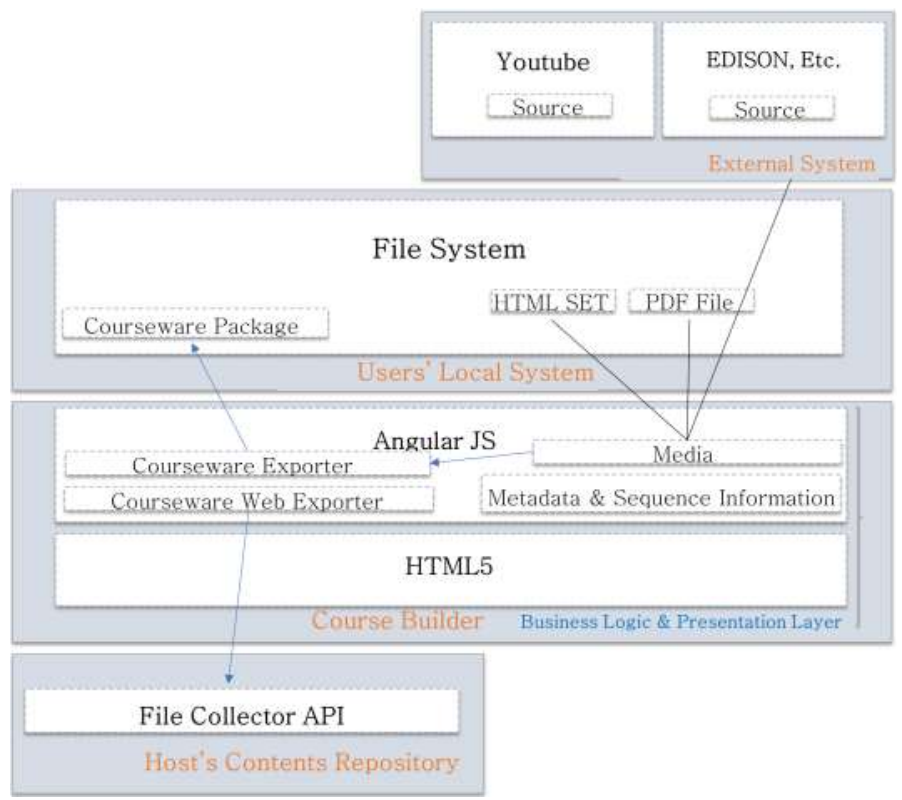

Figure 7. Online Course and Contents for the Authoring System

Currently, EDISON MOOC [7] is developed as an innovative user-interactive online education content by using the above course builder authoring tools with the support of 
Korea Institute of Science and Technology Information (KISTI) and the help of computational fluid dynamics professors from six fields. There are three main characteristics, namely, on-line public classes and e-learning contents based on the professors' fluid dynamics theory lecture videos, interworking with EDISON to provide an interactive educational environment, and finally the provision of a detailed tutorial by teaching assistants.

\subsection{Open Online Course and E-learning Content}

Open online courses with professor's lecture videos are divided into six lectures including computational fluid dynamics introduction, spatial discretization and accuracy, temporal discretization, turbulence modeling, boundary condition, and grid generation. Essentially, it includes the features of online public lectures that make it possible for anyone to freely and repeatedly learn the theoretical knowledge and numerical techniques that are difficult to understand in a class. The unique feature of EDISON MOOC is that as shown in Figure 8 in which e-learning contents including reactive lecture materials and quizzes are provided through the separate screens to improve learner's concentration and understanding.

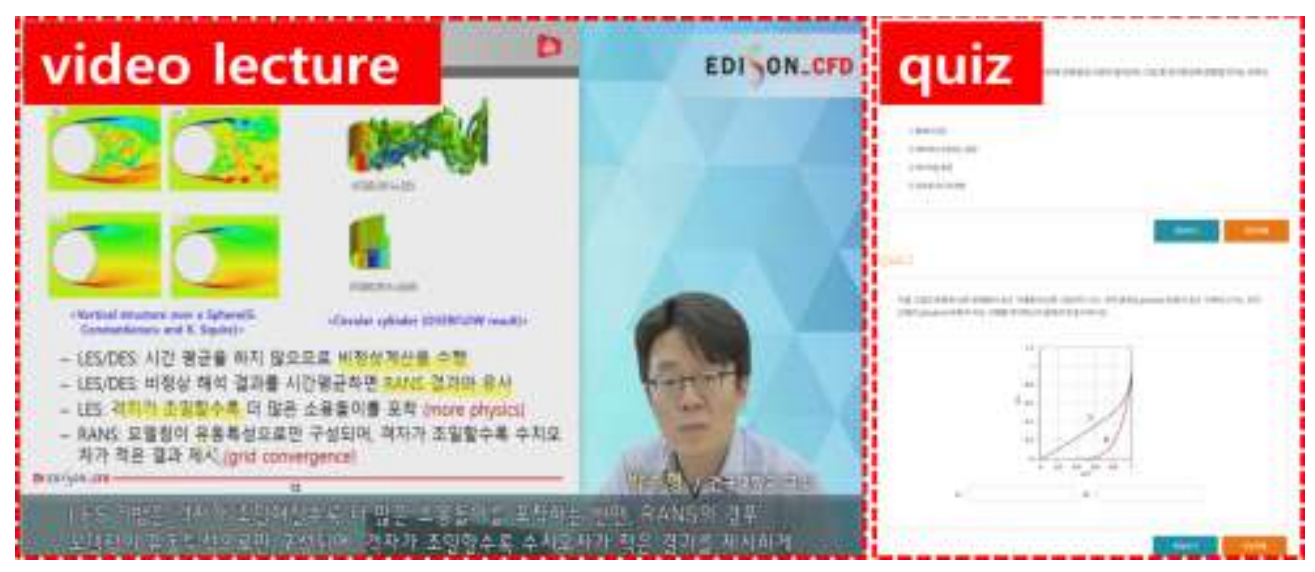

Figure 8. Online Public Lectures and E-Learning Content

\subsection{User-interactive Training Environment}

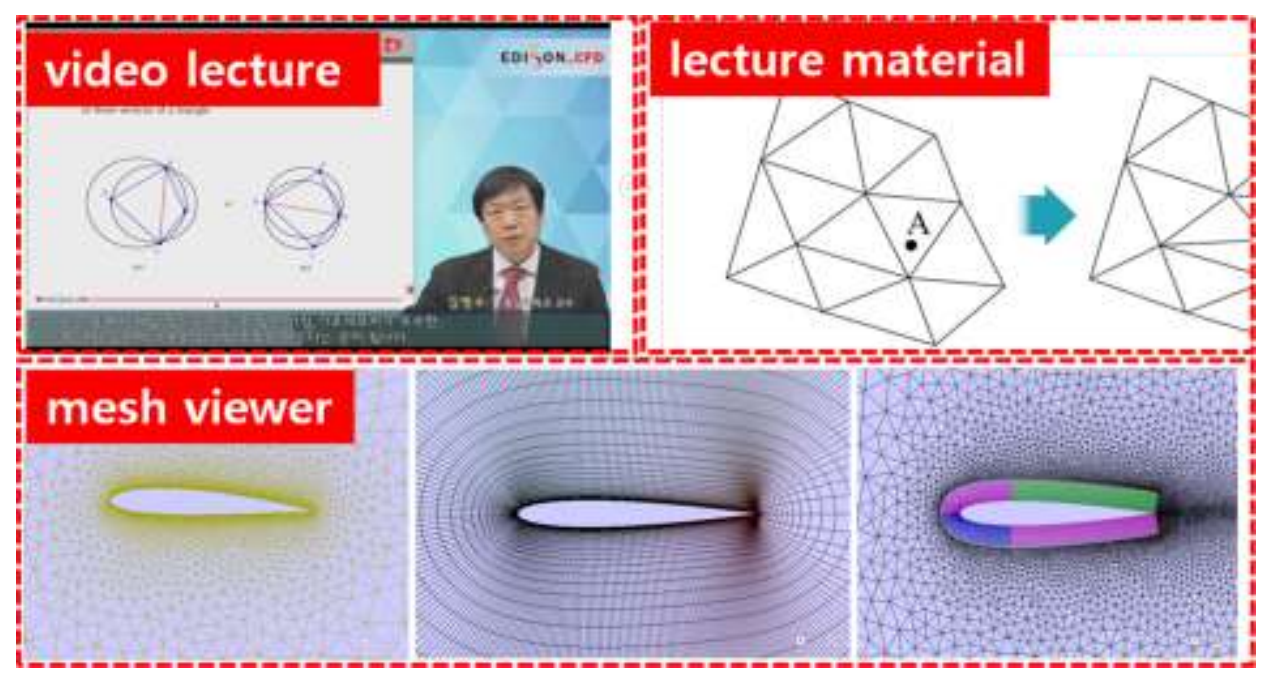

Figure 9. User Interactive Visualization in EDISON MOOC 
Specifically, following its launch in 2011, the EDISON platform has been provides simulation software and contents for computational fluid dynamics on the web. It also provides a computational science simulation framework that makes it easier to understand complex mathematical phenomena and physical phenomena learned in a curriculum. As shown in Figure 9, EDISON MOOC provides a function that allows users to interactively change parameter values or visualize grid and result files through portlets in the middle of online lecture learning by combining these simulation learning environments [8].

\subsection{Practice Description of the Teaching Assistant}

It is not easy for undergraduate students to follow a class by using only lecture materials, and it is very difficult to execute an unfamiliar software using only the manual. EDISON MOOC provides tutorials involving teaching assistants (simulation software developers) to demonstrate background theories and a few analysis cases involving real problems so that it is easy for the students to follow. It is expected that this will immensely aid students in lectures to understand the meaning and utility of a new program, its application in actual engineering fields, and practical implications of the simulation results.

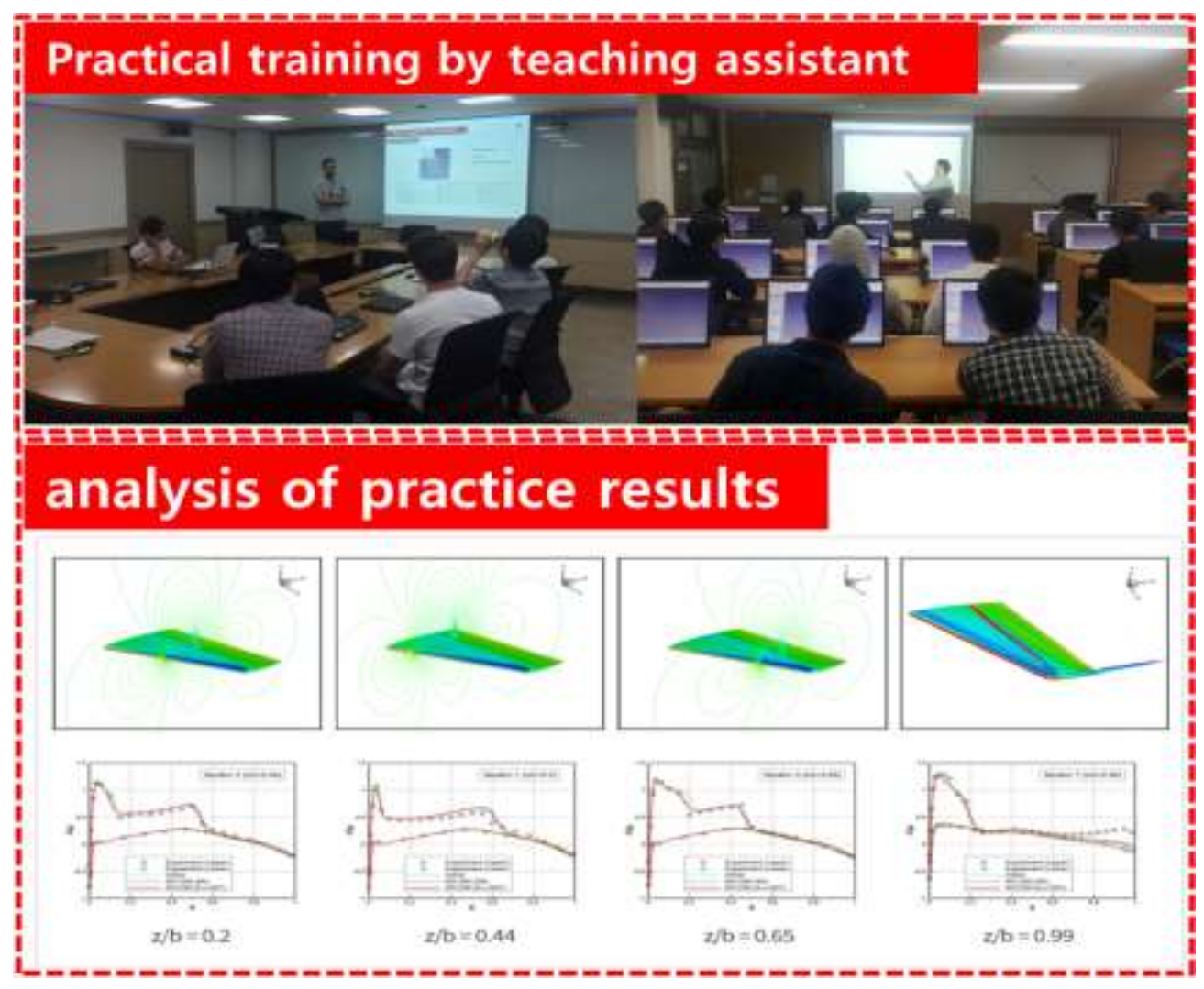

Figure 10. Practical Training by Teaching Assistants

\section{Conclusion}

It is difficult to obtain theoretical knowledge of computational science and engineering, and use unfamiliar tools by only using manuals that lack explanations. Thus, to overcome these difficulties, EDISON's online education system was designed, and EDISON MOOC was developed for the field of computational fluid dynamics, by using free online public lectures. It is structured to increase the concentration and understanding ability of the learners by providing e-learning content including quiz and response lecture materials, which are different from the unilateral lecture method provided by the existing MOOCs. 
Additionally, it provides an intuitive work environment that involves, for example, visualizing the analysis result file directly in the lecture screen or interactively controlling the 3D grid. The construction of a self-sustaining educational ecosystem that utilizes EDISON MOOC with efficiency and qualitative growth in engineering education is expected through shift from lecturer-centered classes to learner-centered creative and active learning.

\section{Acknowledgments}

This paper is a revised and expanded version of a paper entitled [Development of Online Engineering Education System using Web-based Computational Science Platform] presented at [Gi-Myeong Ryu, Jung-Hun Shin, Sik Lee and Kum-Won Cho, Daejeon, Korea, (2017) December 21-23]. And This research was supported by the EDISON Program through the National Research Foundation of Korea (NRF) funded by the Ministry of Science and ICT (No. NRF-2011-0020576).

\section{References}

[1] G.-M. Ryu, J.-H. Shin, S. Lee and K.-W. Cho, "Development of Online Engineering Education System using Web-based Computational Science Platform", Proceedings of the International Journal of Computer Science and Information Technology for Education, Daejeon, Korea, (2017) December 21-23.

[2] EDISON challenge: https://www.edison.re.kr/web/challenge, (accessed January, 2018).

[3] NCN(Network for Computational Nanotechnology): https://nanohub.org/groups/ncn, (accessed January, 2018).

[4] G.-M. Ryu, I. Jeon, J.-H. Shin, S. Lee and K.-W. Cho, "Construction of Paper Repository for Competitive Exhibition using a Web-based Computational Science Platform", Proceedings of the KSII Fall Conference, Seoul, Korea, (2017) November 3-4.

[5] M. Seok Kang, "Status and Directions of Application of MOOCs in View of Education", Proceedings of the KSME Spring Conference, Seoul, Korea, (2015) June 25-26

[6] S. Jung-hun, R. Gi-yeong, J. Cheol-hoon, L. Sik and C. Kum-Won, "Development of Interactive eLearning Authoring Tool for Computational Engineering", Proceedings of the Korea Contents Association, Daejeon, Korea, (2017) May 12-13.

[7] EDISON MOOC: http://mooc.edison.re.kr, (accessed January, 2018)

[8] K. Won Cho, D. Nam, D. Jin, B. Ahn, S. Park, J. Ruth Lee and C. Kim, "Development and Implementation of Simulation based Higher Education-Research Environment", Proceeding of the KSME Fall Conference, Changwon, Korea, (2012) November 7-9.

\section{Authors}

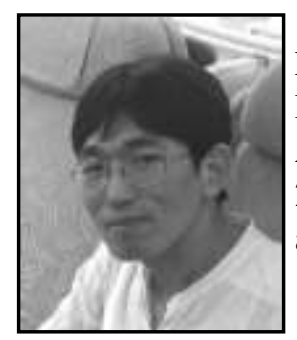

Gi-Myeong Ryu is a Researcher at the Computational Science and Engineering Center of Korea Institute of Science and Technology Information (KISTI), South Korea. He received BEng, MEng degrees in Aerospace Engineering from Chungnam National University in 2013, 2016 respectively. His research interests computational science, aerospace and fluid analysis.

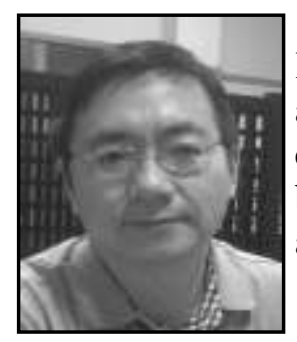

Jung-Hun Shin received BEng, MEng, and DEng degrees at Korea Advanced Institute of Science and Technology in 1999, 2002, and 2013 respectively. His academic degrees were related to computational science and engineering. His industrial interests have been robust and reliable design in various machines such as precision and mechatronic, and medical ones. 


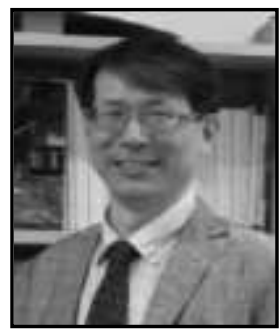

Sik Lee received $\mathrm{Ph} . \mathrm{D}$. in Chemistry from Pohang University of Science and Technology (POSTECH) in 1996 respectively. His academic degrees were related to computational chemistry. $\mathrm{He}$ is a principal researcher and head of Scientific Platform Development of Korea Institute of Science and Technology Information (KISTI), Korea. He joined KISTI in 2000 as a senior.

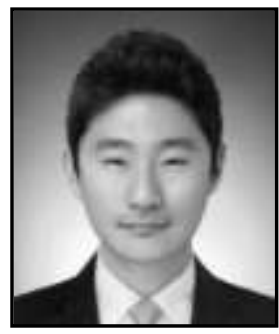

Kum Won Cho received his Ph.D. in Mechanical (Aerospace) Engineering from Korea Advanced Institute of Science and Technology (KAIST) in 2000 respectively. He is a head of Computational Science and Engineering Center, National Institute of Supercomputing and Networking, Korea Institute of Science and Technology Information (KISTI), Korea. 
International Journal of Advanced Science and Technology

Vol.115 (2018) 